\title{
Electromechanical effects on multilayered cells in nonuniform rotating fields
}

\author{
José Luis Sebastián, ${ }^{*}$ Sagrario Muñoz, Miguel Sancho, and Genoveva Martínez \\ Departamento de Física Aplicada III, Facultad de Ciencias Físicas, Universidad Complutense, E-28040 Madrid, Spain \\ Gabriel Álvarez \\ Departamento de Física Teórica II, Facultad de Ciencias Físicas, Universidad Complutense, E-28040 Madrid, Spain
}

(Received 17 May 2011; published 28 July 2011)

\begin{abstract}
We use the Maxwell stress tensor to calculate the dielectrophoretic force and electrorotational torque acting on a realistic four-shelled model of the yeast Saccharomyces cerevisiae in a nonuniform rotating electric field generated by four coplanar square electrodes. The comparison of these results with numerical calculations of the dipolar and quadrupolar contributions obtained from an integral equation for the polarization charge density shows the effect of the quadrupole contribution in the proximity of the electrode plane. We also show that under typical experimental conditions the substitution of the multilayered cell by an equivalent cell with homogeneous permittivity underestimates the quadrupole contribution to the force and torque by 1 order of magnitude.
\end{abstract}

DOI: 10.1103/PhysRevE.84.011926

PACS number(s): 87.50.ch

\section{INTRODUCTION}

Biomedical and biotechnological applications of dielectrophoresis (DEP) and electrorotation (ER) are currently very active areas of research [1]. Since the original works, which dealt only with homogeneous spherical particles [2,3], considerable progress has been made both in theoretical calculations with realistic particle models and colloidal suspensions [4-6] and in the development of practical applications like biosensors and biomicrofluidic platforms [1].

At lowest order in the applied field the dielectrophoretic force on a particle is proportional to the in-phase part of the induced dipole moment and to the gradient of the timeaveraged applied electric field, whereas the electrorotational torque is proportional to the out-of-phase part of the induced dipole moment and to the time-averaged applied electric field [7-10]. Since at this order the induced dipole is itself proportional to the applied field, controlling the square and the gradient of the square of the electric field are critical issues in the design of the electrodes that generate the rotating fields in an ER chamber [1,11-17].

The simplest and most direct approach to calculate the force and the torque is a two-step process known as the effective dipole moment method [18], whereby first one calculates the dipole moment induced in the particle by the electric field and then the force and torque on the particle follow from standard formulas of classical electrodynamics [19]. Note that in this approach multilayered particles have to be replaced by an equivalent homogeneous particle with a radius equal to that of the outermost shell. But even with this replacement, the method does not give accurate results whenever the electric field varies significantly over different layers of the particle with different electrical properties. Moreover, it has been reported that the effective equivalent permittivity that reproduces the dipole moment correctly does not give accurate results for quadrupole and higher multipoles [20].

The preferred approach for realistic cell models with multilayered structures in highly nonuniform fields is a

\footnotetext{
*jlsf@ fis.ucm.es
}

numerical calculation of the Maxwell stress tensor on the surface of the cell, from which the force and torque can be calculated directly by integration of the normal component of the stress and of the cross product of the stress with the radial vector over the entire particle surface respectively [21]. Here the limiting factor is the accuracy of numerical methods (such as the finite element method [22-24]) in the computation of fields in shelled particles with layers of very different thicknesses and electrical properties. Therefore, although this approach has been used extensively in the literature, it has been mostly applied to homogeneous spherical particles in slightly nonuniform electric fields [25-33], and to our knowledge, no work appears to assess the influence of higher order multipole contributions to forces and torques under typical experimental conditions for nonhomogeneous, multilayered particles.

In this paper we calculate the force and torque on a realistic four-shelled model of the yeast Saccharomyces cerevisiae in nonuniform rotating fields of typical ER chambers. Our aim is twofold: to assess quantitatively the effects of high-order multipole contributions and to give an alternative approach to using the same equivalent permittivity that reproduces correctly the dipole contribution in the calculation of the quadrupole contribution.

The layout of the paper is as follows. In Sec. II we first describe the ER chamber and calculate the squared electric field and its gradient with a finite element numerical method. To sample the different regions of field inhomogeneity within the chamber we perform two types of calculations: in the first type we fix the height of the cell with respect to the base of the chamber and increase the electrode gap; in the second, we fix the separation between the electrodes and increase the height of the cell with respect to the base of the chamber. Then we present our model of the yeast and calculate its complex polarizability from the corresponding internal electric field distribution. In Sec. III we present the results for the force and torque obtained with the Maxwell stress tensor and compare them with the corresponding results obtained from the dipolar approximation using the complex polarizability obtained in the previous section. Then we discuss the analytic formulas for the second-order contributions, underlining the fact that these formulas assume a homogeneous particle and therefore require 
the calculation of the permittivity of an equivalent homogenous particle for a multilayered particle. This equivalent permittivity is computed to give the dipole moment correctly but may not give accurate results for the quadrupole contribution, where it enters by way of the second-order Clausius-Mossotti factor. We show that the frequencies at which the force and torque change sign are essentially determined by second-order contributions that can be isolated using the charge density boundary element method (BEM), which takes into account the electrical effects of each individual layer and avoids the approximation of using the permittivity and conductivity of an equivalent homogeneous cell. We discuss our conclusions in Sec. IV.

\section{ELECTROROTATION CHAMBER AND CELL MODEL}

\section{A. Electrorotation chamber}

A variety of chambers with different electrode configurations and correction factors for the field strength are available in the literature [16,34-38], and it is well established that the most homogeneous fields are generated by circular electrodes [16]. Since we are interested in the interaction of particles with the highly nonuniform fields typically produced by electrodes with rectangular corners, in this work we use the planar four-electrode configuration placed on the base of the chamber shown in Fig. 1. The side of the square electrodes has a length $\ell=100 \mu \mathrm{m}$ and the gap between electrodes will range from $\delta=5 \mu \mathrm{m}$ to $\delta=50 \mu \mathrm{m}$. The chamber is filled with a suspending medium of relative permittivity $\varepsilon_{\mathrm{r}}=80$ and conductivity $\sigma=0.12 \mathrm{~S} / \mathrm{m}$.

To generate the rotating field, the electrodes are driven by four sinusoidal voltages of equal amplitude $V=5 \mathrm{~V}$ successively phase-shifted by $\pi / 2[13,39,40]$. We study the frequency range from $10 \mathrm{kHz}$ to $300 \mathrm{MHz}$ and assume that the electrode response is linear, i.e., that no frequency components other than those applied to the electrodes can evolve in the chamber and that electrode polarization effects are negligibly small. In addition, we do not consider in our calculations the influence of the gradient of the phase of the electric field on the force and torque, which could produce traveling wave effects [11].

The cell model used in this study has a diameter of approximately $6 \mu \mathrm{m}$ and will always be placed on the vertical

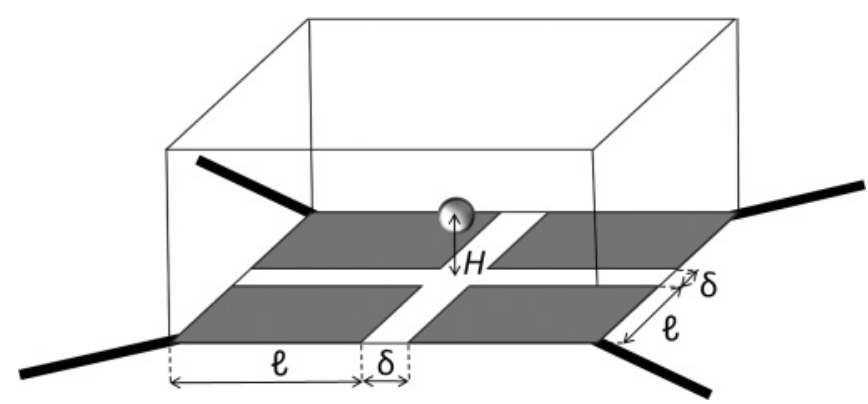

FIG. 1. (Color online) Electrorotation chamber with four square electrodes of side length $\ell$ and gap $\delta$ on the base. The chamber is filled with a suspending medium with $\varepsilon_{\mathrm{r}}=80$ and $\sigma=0.12 \mathrm{~S} / \mathrm{m}$. The figure shows also a cell (not to scale) placed at a height $H$ above the electrode plane. symmetry axis of the chamber with the cell center at heights $H$ from 3 to $14 \mu \mathrm{m}$ above the electrode plane. To calculate the electric field distribution to which the cell will be exposed in this chamber, we first solved the Laplace equation (with the suspending medium but without the cell) using the finite element analysis software COMSOL Multiphysics. In Fig. 2 we show the squared electric field and (minus) its gradient along the vertical axis as a function of $H$ for four values of the gap, from top to bottom $\delta=5,10,20$, and $50 \mu \mathrm{m}$, respectively. The frequency is $1 \mathrm{MHz}$. Arrows in Fig. 2(b) mark the height at which each maximum is reached, which is an increasing function of the gap $\delta$. In fact the maximum for $\delta=50 \mu \mathrm{m}$ is located at $H=18 \mu \mathrm{m}$, off the horizontal scale.

Figure 2 gives a first indication to understand the physical behavior of the system, because we recall that in the dipole approximation [cf. Eqs. (3) and (4)], and ignoring momentarily the effect of the particle on the field, the torque is essentially the imaginary part of the particle effective polarizability times the magnitude shown in Fig. 2(a), while the force is essentially the real part of the effective polarizability times the magnitude shown in Fig. 2(b). We next address the calculation of this polarizability.

\section{B. Polarizability of the yeast cell}

The yeast $S$. cerevisiae has been extensively studied and multiple DEP and ER spectra of single yeast cells have been measured at different medium conductivities. Although initially double-shelled models were used for theoretical calculations [41-43], only the extension to four shells rendered a good agreement with experimental ER spectra. In fact, electron micrographs of $S$. cerevisiae reveal a three-layered structure outside the plasma membrane [44-46]. Therefore in this work we use the complete spherical four-shelled model (cell interior, plasma membrane, thick inner wall, and thin outer wall) that best fits the experimental data [43]. The geometrical and electrical parameters of this model are given in Table I.

As our first use of this model we calculate its complex effective polarizability $\tilde{\alpha}_{\text {eff }}$, which gives the relation between the inducing external field at the center of the cell $\widetilde{\mathbf{E}}_{0}$ and the effective induced dipole moment

$$
\tilde{\mathbf{p}}_{\text {eff }}=\tilde{\alpha}_{\mathrm{eff}} \widetilde{\mathbf{E}}_{0}
$$

later to be substituted in the dipole approximation, Eqs. (3) and (4). In Ref. [47] we showed that the effective polarizability of a particle consisting of $N$ layers and immersed in an external medium can be determined by the equation

$$
\tilde{\alpha}_{\mathrm{eff}} \widetilde{\mathbf{E}}_{0}=\frac{\operatorname{Re}\left(\tilde{\varepsilon}_{\mathrm{ext}}\right)}{\tilde{\varepsilon}_{\mathrm{ext}}} \sum_{i=1}^{N} \int_{V_{i}}\left(\tilde{\varepsilon}_{i}-\tilde{\varepsilon}_{\mathrm{ext}}\right) \widetilde{\mathbf{E}}_{i} d V_{i},
$$

where $\tilde{\varepsilon}_{\text {ext }}=\varepsilon_{\text {ext }}-i \sigma_{\text {ext }} / \omega$ is the complex permittivity of the external medium and where $\tilde{\varepsilon}_{i}, \widetilde{\mathbf{E}}_{i}$, and $d V_{i}$ denote the complex permittivity, electric field distribution, and volume element of the $i$ th particle layer, respectively. Incidentally, we recall that the two factors $\varepsilon_{0}\left(\tilde{\varepsilon}_{i}-\tilde{\varepsilon}_{\text {ext }}\right) / \tilde{\varepsilon}_{\text {ext }}$ and $\operatorname{Re}\left(\tilde{\varepsilon}_{\text {ext }}\right) / \varepsilon_{0}$ must be included in the definition of $\tilde{\mathbf{p}}_{\text {eff }}$ via Eq. (2). The first factor can be physically understood from the analogy of a body immersed in a fluid under a gravitational field: to calculate 

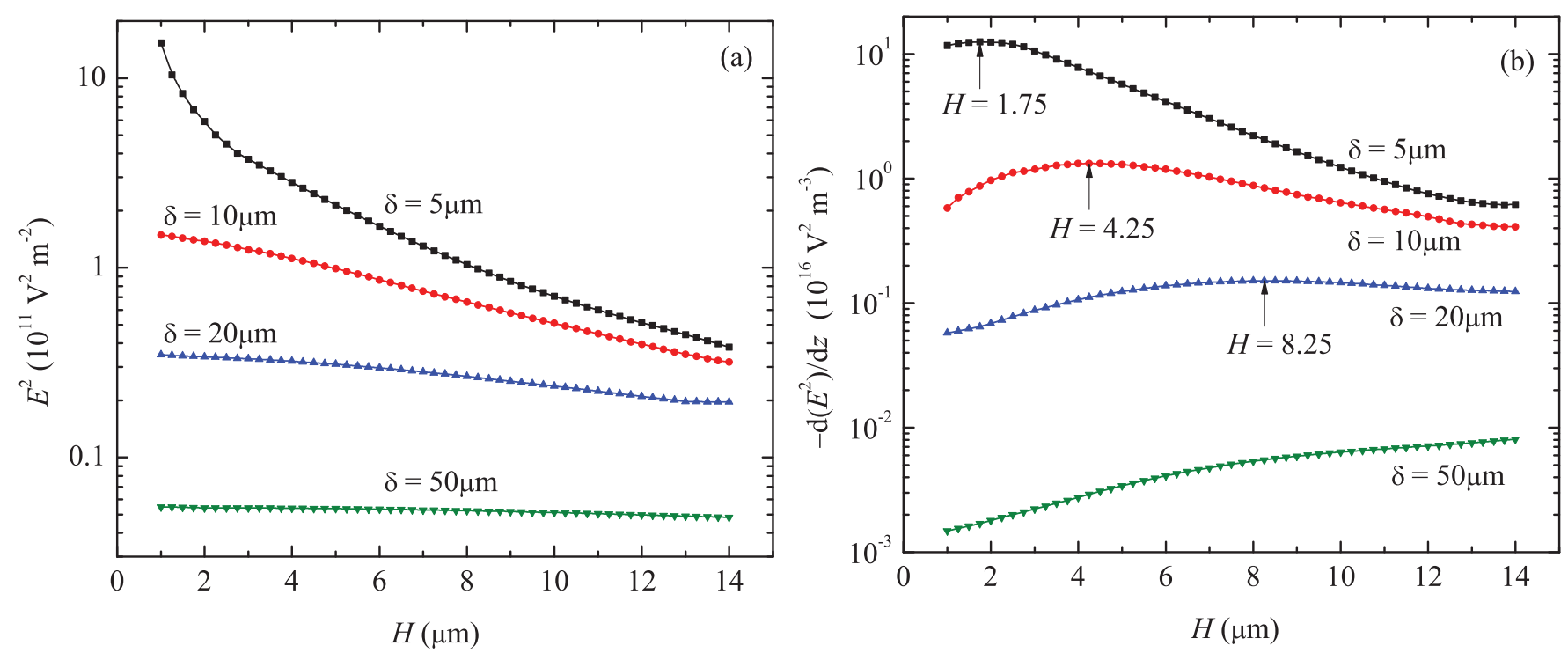

FIG. 2. (Color online) (a) Squared electric field and (b) its gradient along the vertical axis in the chamber as a function of the height $H$ for four values of the gap $\delta$. The frequency is $1 \mathrm{MHz}$. Arrows mark the heights corresponding to the respective maxima. The maximum for $\delta=50 \mu \mathrm{m}$ is located at $H=18 \mu \mathrm{m}$, off the horizontal scale.

the net force we must subtract a "buoyancy term." The second factor is needed because the equivalent charges and dipole are considered to be in vacuum, and therefore the external field has to be multiplied by the relative inductive factor between vacuum and medium.

Following the numerical procedure outlined in Ref. [47] we have calculated the complex polarizability of the yeast model as a function of the frequency $f$ of the applied field and show the results in Fig. 3. In particular, the figure illustrates the well-known fact that the crossover frequency of the ER spectrum (marked with an arrow) is equal to the frequency of the maximum DEP force. Note that due to the frequency range above $10^{4} \mathrm{~Hz}$ and to the low membrane conductivity $\left(10^{-7} \mathrm{~S} / \mathrm{m}\right)$, these results pertain to the so-called $\beta$-relaxation region and typically have an error of less than $1.5 \%$. We mention that, depending on the values of the membrane and bound charge conductivities, surface charges responsible for the membrane potential in live cells can produce important deviations in the $\alpha$-relaxation region. To account for this effect, Prodan et al. [48] gave analytic solutions for a model that includes weak surface charge distributions on low-conductivity membranes, and subsequently Di Biasio et al. [49,50] found a correction term that removes these limitations in the parameter range.

TABLE I. Electrical and geometrical parameters of the fourshelled yeast cell model from Ref. [43].

\begin{tabular}{lcrc}
\hline \hline Region & Radius $(\mu \mathrm{m})$ & $\varepsilon_{\mathrm{r}}$ & $\sigma(\mathrm{S} / \mathrm{m})$ \\
\hline Interior & 2.500 & 51 & 1 \\
Membrane & 2.508 & 5 & $10^{-7}$ \\
Inner wall & 2.708 & 60 & 0.01 \\
Outer wall & 2.758 & 6 & 0.02 \\
\hline \hline
\end{tabular}

\section{FORCE AND TORQUE RESULTS}

\section{A. Maxwell stress tensor and dipole approximation results}

We denote by $\mathbf{F}$ and $\mathbf{N}$ the force and torque obtained from a numerical integration of the Maxwell stress tensor over the particle surface, and by $\mathbf{F}^{(1)}$ and $\mathbf{N}^{(1)}$ the first-order, dipole approximation force and torque obtained from the complex effective polarizability derived via Eq. (2). The latter expressions can be found, e.g., in Ref. [25] and read

$$
\begin{gathered}
\mathbf{F}^{(1)}=\frac{1}{4} \operatorname{Re}\left[\left(\widetilde{\mathbf{p}}_{\text {eff }} \cdot \nabla\right) \widetilde{\mathbf{E}}_{0}^{*}\right]=\frac{1}{4} \operatorname{Re}\left(\tilde{\alpha}_{\text {eff }}\right) \nabla\left(E_{0}^{2}\right), \\
\mathbf{N}^{(1)}=\frac{1}{2} \operatorname{Re}\left(\widetilde{\mathbf{p}}_{\text {eff }} \times \widetilde{\mathbf{E}}_{0}^{*}\right)=-\frac{1}{2} \operatorname{Im}\left(\tilde{\alpha}_{\text {eff }}\right) E_{0}^{2} \mathbf{e}_{z} .
\end{gathered}
$$

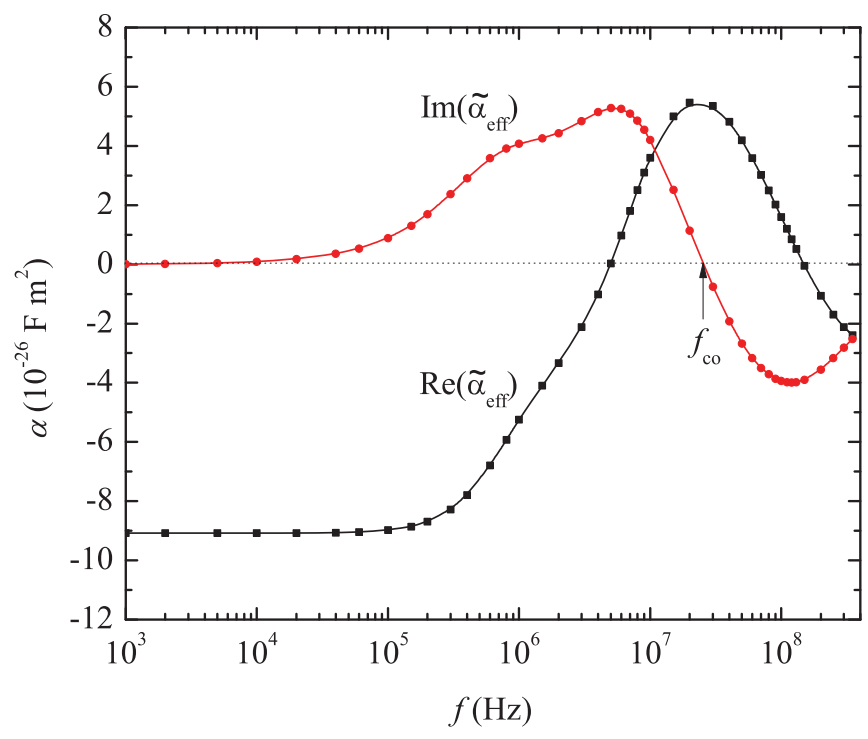

FIG. 3. (Color online) Real and imaginary parts of the effective complex polarizability $\tilde{\alpha}_{\text {eff }}$ of the yeast model described in Table I as a function of the frequency $f$ of the applied field. The arrow marks the crossover frequency $f_{\mathrm{co}}$. 

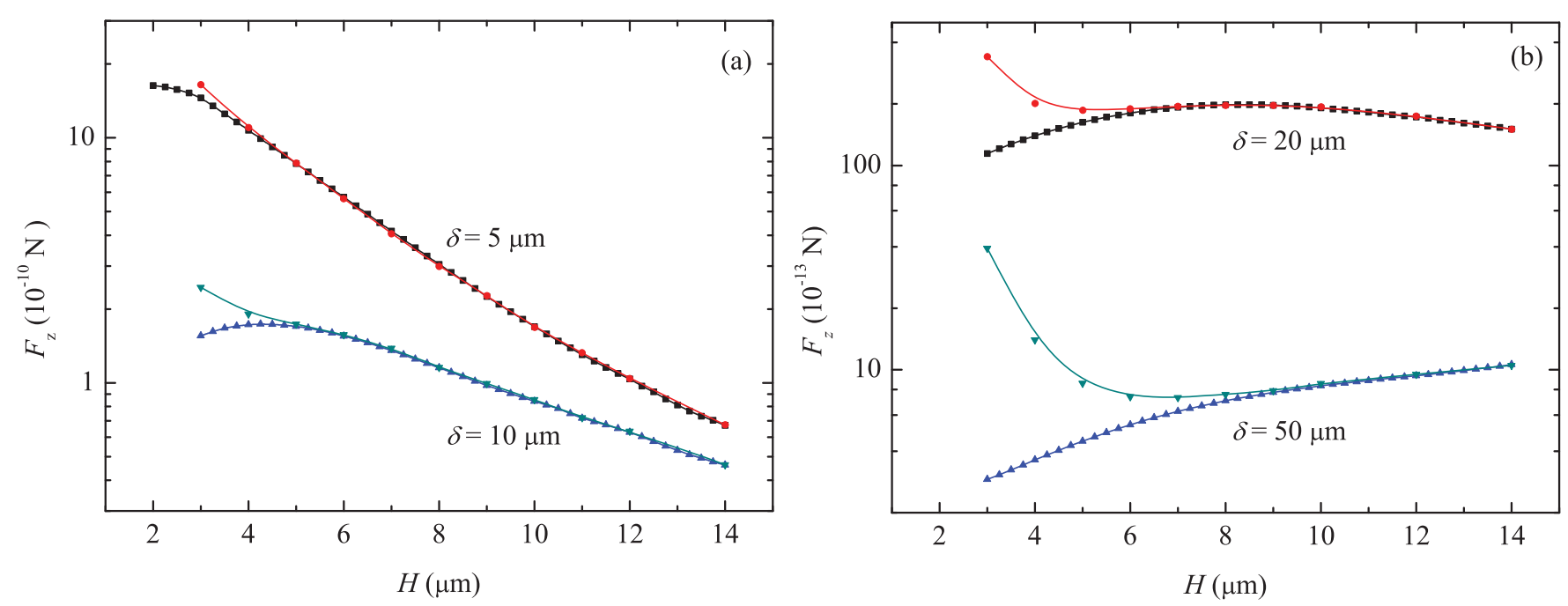

FIG. 4. (Color online) Total force from the Maxwell stress tensor (upper curve of each pair) and dipolar component from Eq. (3) (lower curve of each pair) on the yeast cell for a frequency of $1 \mathrm{MHz}$ as a function of the height $H$ for four values of the electrode gap $\delta$. Note the difference in the vertical scales in (a) and (b).

Incidentally, note that in the case of multilayered cells, this approach bypasses the iterative calculation of an equivalent homogeneous particle with an equivalent permittivity. (We show later that both methods agree to a remarkable precision.)

Figure 4 shows the forces $F_{z}$ and $F_{z}^{(1)}$ on the yeast cell as a function of the height $H$ for the same four electrode gaps and frequency as in Fig. 2. We recall that the radius of the particle (i.e. the minimum height at which it can be placed) is approximately $3 \mu \mathrm{m}$. The part of the $F_{z}^{(1)}$ curve that extends below this value in Fig. 4(a) has been extrapolated as if it were a "point dipole" to show the effect of the corresponding maximum in Fig. 2(b). If the electrode gap $\delta$ is small (comparable to the particle diameter), the dipole approximation quickly approaches the Maxwell stress tensor result, and only when the cell is very close to the electrode plane are higher order contributions and image effects from the electrodes noticeable. However, as the gap widens and the maximum of the $-\nabla\left(E_{0}^{2}\right)$ in Fig. 2(b) moves to the right, the differences between the two results increase.

Figure 5 shows similar results for the torque. For the highest values of the $E^{2}$ profile corresponding to the smallest gap in Fig. 2(a), the contribution of higher order terms to the total torque is very small. As the gap is widened, the electric field is reduced and higher order contributions become significant for heights up to the diameter of the cell.

In Fig. 6 we analyze the influence of quadrupole and higher order contributions in the electrokinetic behavior of the yeast cell by comparing the DEP and ER spectra in the range from $10 \mathrm{kHz}$ to $300 \mathrm{MHz}$ for a fixed electrode gap $\delta=20 \mu \mathrm{m}$ and two positions of the cell in the chamber, $H=4 \mu \mathrm{m}$ and $H=8 \mu \mathrm{m}$. Each spectrum is calculated twice: first with the accurate Maxwell stress tensor method and then
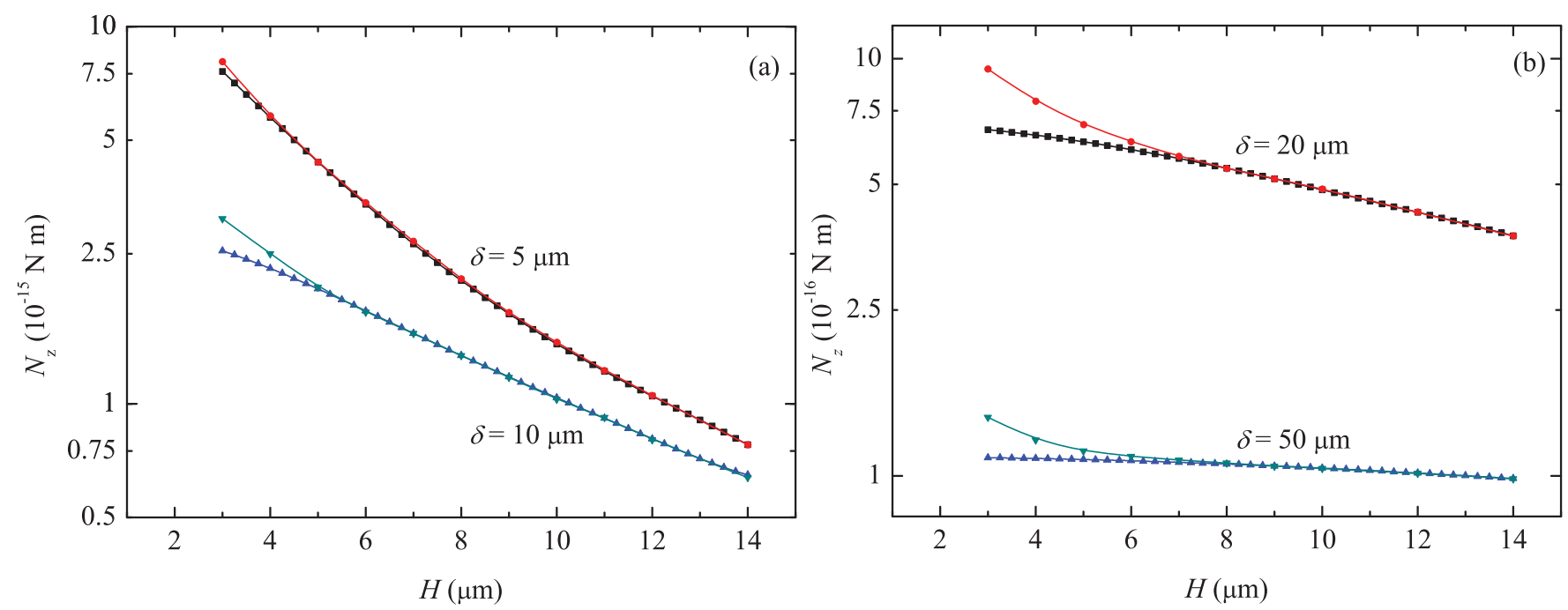

FIG. 5. (Color online) Total torque from the Maxwell stress tensor (upper curve of each pair) and dipolar component from Eq. (4) (lower curve of each pair) on the yeast cell for a frequency of $1 \mathrm{MHz}$ as a function of the height $H$ for four values of the electrode gap $\delta$. Note the difference in the vertical scales in (a) and (b). 

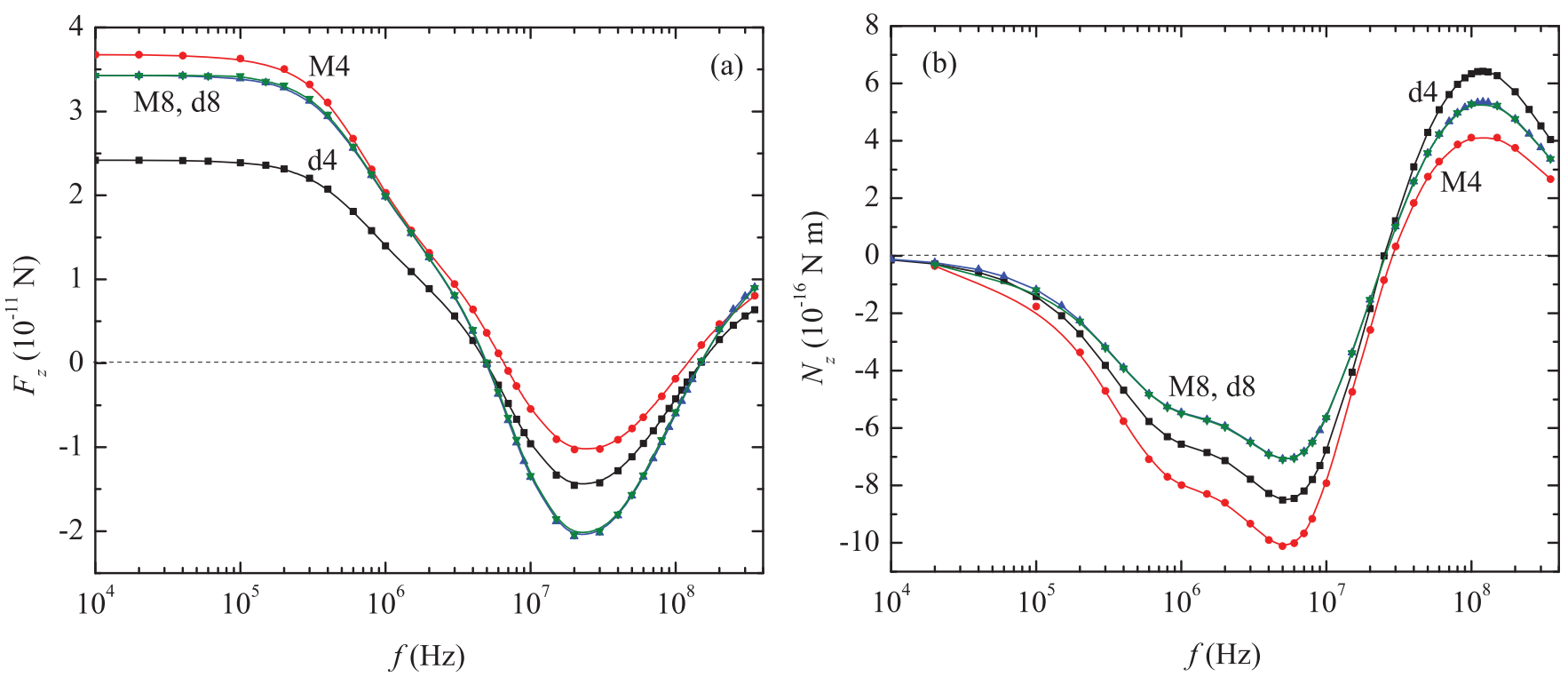

FIG. 6. (Color online) (a) DEP and (b) ER spectra for a fixed gap $\delta=20 \mu \mathrm{m}$ : (M8; $\nabla$ ) Maxwell stress tensor results at $H=8 \mu \mathrm{m}$; (d8; $\triangle$ ) dipole approximation at $H=8 \mu \mathrm{m}$; (M4) Maxwell stress tensor results at $H=4 \mu \mathrm{m}$; and (d4) dipole approximation at $H=4 \mu \mathrm{m}$. For $H=8 \mu \mathrm{m}$ higher order terms are negligible and the corresponding markers and curves appear to be almost superimposed.

with the dipole approximation given by Eqs. (3) and (4). If the cell is far from the electrodes $(H=8 \mu \mathrm{m})$, higher order terms have a negligible influence on either the force or the torque, to the extent that the corresponding markers and curves appear superimposed in Fig. 6. However, when the cell is close to the electrodes $(H=4 \mu \mathrm{m})$ the crossover frequencies for the force and torque in the more accurate Maxwell stress tensor spectra are shifted toward higher values with respect to the corresponding values for the dipole analysis, $f_{\mathrm{DEP}}=5 \mathrm{MHz}$ and $f_{\mathrm{ER}}=25 \mathrm{MHz}$. Therefore these crossover frequency shifts should be due mainly to the quadrupole and higher contributions included in the Maxwell stress tensor approach. We confirm this assumption in the next section.

\section{B. Analytic expressions for the quadrupole contribution}

There are analytic expressions for the second-order contribution to the force and the torque, but all the formulas we are aware of are valid only for homogeneous particles. To apply them to our four-shelled particle, in the next section we calculate the complex permittivity $\tilde{\varepsilon}_{\text {eq }}$ of a homogeneous equivalent sphere with the same radius as our yeast model and, as a first consistency check, compare the results obtained with our previous calculation using $\tilde{\alpha}_{\text {eff }}$, Eqs. (3) and (4), with the corresponding first-order force and torque reported in the literature $[1,21]$,

$$
\begin{aligned}
& \mathbf{F}^{(1)}=\pi R^{3} \operatorname{Re}\left(\tilde{\varepsilon}_{\text {ext }}\right) \operatorname{Re}\left(f_{\mathrm{CM}}\right) \nabla\left(E_{0}^{2}\right), \\
& \mathbf{N}^{(1)}=2 \pi R^{3} \operatorname{Re}\left(\tilde{\varepsilon}_{\text {ext }}\right) \operatorname{Im}\left(f_{\mathrm{CM}}\right) E_{0}^{2} \mathbf{e}_{z},
\end{aligned}
$$

where $f_{\mathrm{CM}}$ is the Clausius-Mossotti factor,

$$
f_{\mathrm{CM}}=\frac{\tilde{\varepsilon}_{\mathrm{eq}}-\tilde{\varepsilon}_{\mathrm{ext}}}{2 \tilde{\varepsilon}_{\mathrm{eq}}+\tilde{\varepsilon}_{\mathrm{ext}}} .
$$

In Appendix F of Ref. [25], Jones uses general multipolar theory (i.e., without the linear multipole approximation) to derive the analytical expressions for the second-order force and torque on a homogeneous dielectric sphere, including the expressions of the Clausius-Mossotti factors that are valid in this context. In the notation of Ref. [21] these analytical expressions are

$$
\begin{gathered}
\mathbf{F}^{(2)}=\operatorname{Re}\left(\widetilde{K}^{(2)}\right) \sum_{i, j, k} \partial_{k}\left(\partial_{i} E_{j}\right)^{2} \mathbf{e}_{k}, \\
\mathbf{N}^{(2)}=2 \operatorname{Im}\left(\widetilde{K}^{(2)}\right) \operatorname{Im}\left[\sum_{i, j, k, p} \epsilon_{i j k}\left(\partial_{p} E_{0 i}^{*}\right)\left(\partial_{p} E_{0 j}\right) \mathbf{e}_{k}\right],
\end{gathered}
$$

where the indices are summed over the components,

$$
\widetilde{K}^{(2)}=\frac{\pi}{3} R^{5} \operatorname{Re}\left(\tilde{\varepsilon}_{\text {ext }}\right) f_{\mathrm{CM}}^{(2)},
$$

and $f_{\mathrm{CM}}^{(2)}$ is the second-order Clausius-Mossotti factor,

$$
f_{\mathrm{CM}}^{(2)}=\frac{2\left(\tilde{\varepsilon}_{\mathrm{eq}}-\tilde{\varepsilon}_{\mathrm{ext}}\right)}{2 \tilde{\varepsilon}_{\mathrm{eq}}+3 \tilde{\varepsilon}_{\mathrm{ext}}} .
$$

Note, however, the structure of Eqs. (8) and (9), where $\widetilde{K}^{(2)}$ multiplies terms that represent the spatial variation of the electric field. The factor $\widetilde{K}^{(2)}$ includes all the electrical and geometrical information pertaining to the cell and medium, and the question arises whether the second-order ClausiusMossotti factor (which gives the correct result for an initially homogeneous particle) gives an accurate result for the initially nonhomogeneous particle using only the $\tilde{\varepsilon}_{\mathrm{eq}}$ that reproduces correctly first-order effects. In fact, we will show that the smeared-out equivalent permittivity that reproduces the dipole moment of a multishelled particle does not reproduce the quadrupole contribution correctly. We address this issue in the next section, where we calculate numerically a $\widetilde{K}^{(2)}$ for the actual multilayered model by using the BEM, a numerical technique that is more suitable to isolate the quadrupole contribution than the finite element method. 


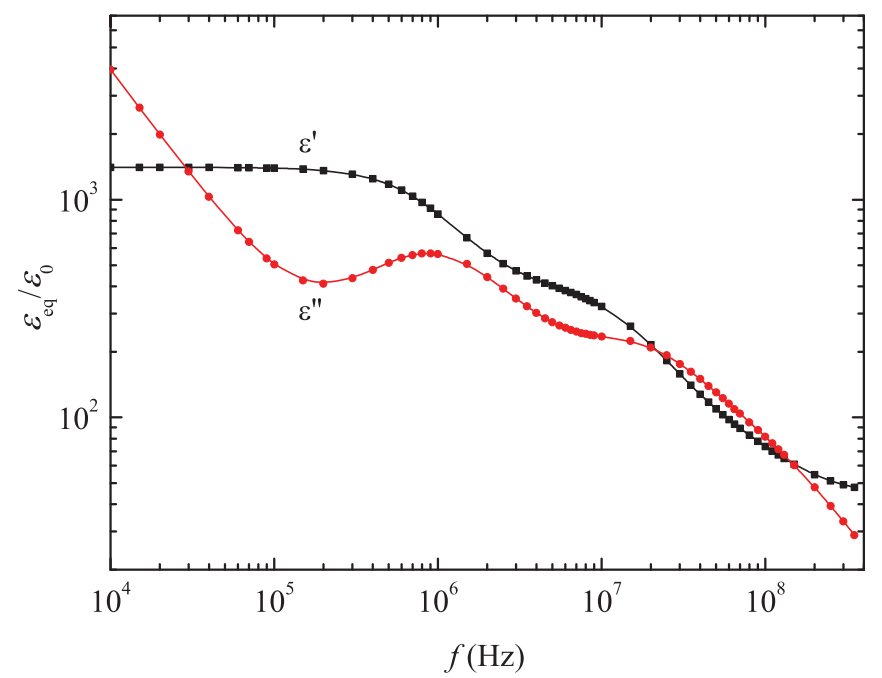

FIG. 7. (Color online) Relative real part $\varepsilon^{\prime}$ and relative imaginary part $\varepsilon^{\prime \prime}$ of the complex permittivity $\tilde{\varepsilon}_{\text {eq }}=\varepsilon_{0}\left(\varepsilon^{\prime}-i \varepsilon^{\prime \prime}\right)$ of an equivalent homogeneous sphere as a function of the frequency $f$ of the external field.

\section{Equivalent permittivity and second-order contribution from the boundary element method}

First, we check that our numerical computation of $\tilde{\alpha}_{\text {eff }}$ agrees with the results given by an equivalent permittivity model. To calculate $\tilde{\varepsilon}_{\text {eq }}$ we start with the innermost sphere and the layer enclosing it, then repeat the procedure on the new, now homogeneous particle and the layer enclosing it, and continue until the outermost shell has been incorporated. The results are shown in Fig. 7. Comparing Eqs. (3) and (4) to Eqs. (5)-(7), we should find that up to numerical errors $\operatorname{Re}\left(\tilde{\alpha}_{\text {eff }}\right)=4 \pi R^{3} \operatorname{Re}\left(\tilde{\varepsilon}_{\text {ext }}\right) \operatorname{Re}\left(f_{\mathrm{CM}}\right)$, and $\operatorname{Im}\left(\tilde{\alpha}_{\text {eff }}\right)=-4 \pi R^{3} \operatorname{Re}\left(\tilde{\varepsilon}_{\text {ext }}\right) \operatorname{Im}\left(f_{\mathrm{CM}}\right)$. The results agree with a relative error of less than $10^{-5}$, thus confirming that the equivalent homogenous particle approach gives the first-order effects for a multilayered particle accurately.

To calculate the second-order contributions to the force and torque we use a technique that, although belonging to the family of BEMs in that only bounding surfaces have to be discretized, is based on an integral equation for the polarization charge density induced on the dielectric interfaces in the quasistatic approximation [51,52]. Since the numerical discretization of this integral equation does not involve any type of polynomial approximation, the surface mesh can consist of a reasonably small number of elements that yield an easily solvable system of linear equations free from numerical instabilities (contrary to what happens in the discretization of similar integral equations for the electric potential for a current source [53]). As an additional advantage, the method gives physical insight into the induced charges and interfacial relaxation mechanisms that take place along the frequency spectrum. We give a brief account of the method here and refer to Ref. [51] for further details.

When a homogeneous particle of complex permittivity $\tilde{\varepsilon}_{1}$ is immersed in a linear dielectric medium of complex permittivity $\tilde{\varepsilon}_{2}$ where an AC field exists, the net complex charge density at the interface is given by [47]

$$
\tilde{\tau}=\varepsilon_{0} \frac{\tilde{\varepsilon}_{2}-\tilde{\varepsilon}_{1}}{\tilde{\varepsilon}_{1}} \widetilde{E}_{12},
$$

where $\widetilde{E}_{12}$ denotes the normal component of the field directed from medium 1 to medium 2. Equation (12) is a generalization for the corresponding expression for the polarization charge density at the interface between two lossless media. The real part of the complex density $\tilde{\tau}$ includes both the polarization charges produced by the different dipole densities induced by the field at both sides of the interface and the free charges built up at the surface as a consequence of the different conductivities of both media. In Ref. [51] it is shown that the complex surface density $\tilde{\tau}$ satisfies the integral equation

$$
\begin{aligned}
\tilde{\tau}(\mathbf{r})= & 2 \frac{\tilde{\varepsilon}_{1}-\tilde{\varepsilon}_{2}}{\tilde{\varepsilon}_{1}+\tilde{\varepsilon}_{2}} \tilde{\tau}_{0}(\mathbf{r}) \\
& -\frac{1}{2 \pi} \frac{\tilde{\varepsilon}_{1}-\tilde{\varepsilon}_{2}}{\tilde{\varepsilon}_{1}+\tilde{\varepsilon}_{2}} \int_{S} \tilde{\tau}\left(\mathbf{r}^{\prime}\right) \frac{\partial}{\partial n}\left(\frac{1}{\left|\mathbf{r}-\mathbf{r}^{\prime}\right|}\right) d S^{\prime},
\end{aligned}
$$

where $\tilde{\tau}_{0}(\mathbf{r})=\varepsilon_{0} \widetilde{E}_{0 n}(\mathbf{r}), \widetilde{E}_{0 n}(\mathbf{r})$ is the normal component of the field produced by the external sources, and $S$ is the interface. The generalization of Eq. (13) to the case where the particle has several dielectric interfaces is

$$
\begin{aligned}
\tilde{\tau}(\mathbf{r})= & 2 \frac{\tilde{\varepsilon}_{i}-\tilde{\varepsilon}_{j}}{\tilde{\varepsilon}_{i}+\tilde{\varepsilon}_{j}} \tilde{\tau}_{0}(\mathbf{r})-\frac{1}{2 \pi} \frac{\tilde{\varepsilon}_{i}-\tilde{\varepsilon}_{j}}{\tilde{\varepsilon}_{i}+\tilde{\varepsilon}_{j}} \\
& \times \sum_{k} \int_{S_{k}} \tilde{\tau}\left(\mathbf{r}^{\prime}\right) \frac{\partial}{\partial n}\left(\frac{1}{\left|\mathbf{r}-\mathbf{r}^{\prime}\right|}\right) d S^{\prime},
\end{aligned}
$$

where $\mathbf{r}$ is a point at the interface between medium $i$ and medium $j$ and the sum in the last term extends to all the interfaces $S_{k}$.

Equation (14) is a Fredholm integral equation of the second kind for $\tilde{\tau}(\mathbf{r})$. To solve it numerically we divide each surface $S_{k}$ into $n_{k}$ small elements $\Delta_{1}, \ldots, \Delta_{n_{k}}$ and assume $\tilde{\tau}(\mathbf{r})$ to be constant on each of these elements. The integral equation is therefore approximated by a system of linear equations,

$$
\tilde{\tau}_{i}=\sum_{j} A_{i j} \tilde{\tau}_{j}+B_{i},
$$

where

$$
\begin{gathered}
A_{i j}=-\frac{1}{2 \pi} \frac{\tilde{\varepsilon}_{k}-\tilde{\varepsilon}_{l}}{\tilde{\varepsilon}_{k}+\tilde{\varepsilon}_{l}} \int_{\Delta_{j}} \frac{\partial}{\partial n}\left(\frac{1}{\left|\mathbf{r}_{i}-\mathbf{r}^{\prime}\right|}\right) d S^{\prime}, \\
B_{i}=2 \frac{\tilde{\varepsilon}_{k}-\tilde{\varepsilon}_{l}}{\tilde{\varepsilon}_{k}+\tilde{\varepsilon}_{l}} \tilde{\tau}_{0}\left(\mathbf{r}_{i}\right),
\end{gathered}
$$

the point $\mathbf{r}_{i}$ is in the element $\Delta_{i}$, and $\tilde{\varepsilon}_{k}$ and $\tilde{\varepsilon}_{l}$ are the permittivities of the media separated by $\Delta_{i}$. Note that physically $A_{i j}$ is proportional to the normal component of the electric field created at $\mathbf{r}_{i}$ by a uniform charge distribution on the element $\Delta_{j}$. Once we know the charge densities $\tilde{\tau}_{i}=\tilde{\tau}\left(\mathbf{r}_{i}\right)$ the induced dipole moment can be calculated as

$$
\tilde{\mathbf{p}}=\sum_{i} \tilde{\tau}_{i} \mathbf{r}_{i} \Delta_{i},
$$

where we have denoted by the same symbol $\Delta_{i}$ the surface element and its area. 

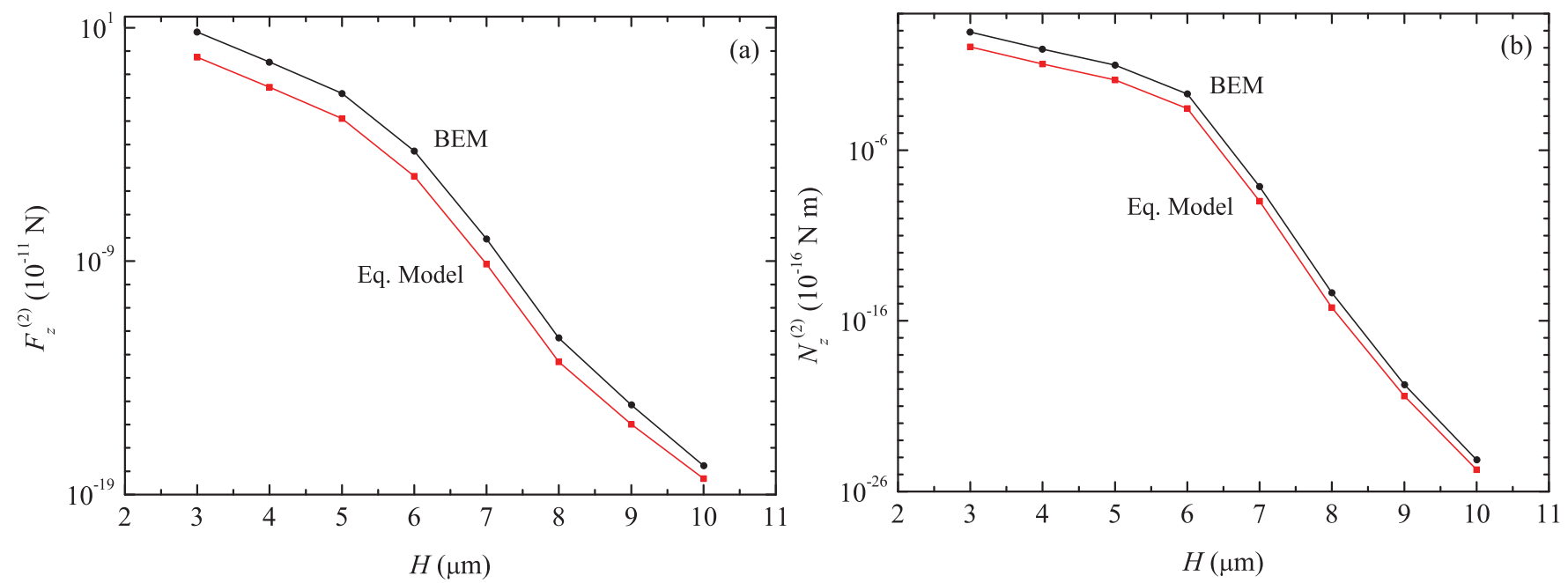

FIG. 8. (Color online) (a) Second-order contributions to the force at the crossover frequency $f=5 \mathrm{MHz}$ using the BEM results and the equivalent model. (b) Second-order contributions to the torque at the crossover frequency $f=25 \mathrm{MHz}$ using the BEM results and the equivalent model.

The quadrupolar moment along the $z$ axis computed from the complex charge density $\tilde{\tau}_{i}$ is

$$
\tilde{p}_{z z}^{(2)}=\sum_{i} \tilde{\tau}_{i} z_{i}^{2} \Delta_{i},
$$

and the real and imaginary parts of $\widetilde{K}_{\mathrm{BEM}}^{(2)}$ can be obtained from [25]

$$
\tilde{p}_{z z}^{(2)}=\widetilde{K}_{\mathrm{BEM}}^{(2)} \frac{\partial E_{z}}{\partial z}
$$

The comparison between the second-order effects for the equivalent [i.e., with $\widetilde{K}^{(2)}$ ] and actual [i.e., with $\widetilde{K}_{\mathrm{BEM}}^{(2)}$ ] models as a function of the cell position $H$ is shown in Fig. 8. This comparison is made at the corresponding crossover frequencies, namely, $f=5 \mathrm{MHz}$ for the force and $f=25 \mathrm{MHz}$ for the torque. The results show that both $F_{z}^{(2)}$ and $N_{z}^{(2)}$ decrease very rapidly with the distance $H$ to the base of the chamber and are negligible at $H=6 \mu \mathrm{m}$ and $H=7 \mu \mathrm{m}$, respectively. These estimates are in very good agreement with the results in Figs. 4 and 5.

We also see that at $H=4 \mu \mathrm{m}$ and $f=5 \mathrm{MHz}$ the BEM method gives for the second-order force a value $F_{z}^{(2)}=$ $0.337 \times 10^{-11} \mathrm{~N}$, whereas for the equivalent model we get an analytical value $F_{z}^{(2)}=0.028 \times 10^{-11} \mathrm{~N}$. Note that the BEM force value is very close to the difference between the total force (from the Maxwell stress tensor) and the dipolar approximation $\Delta F_{z}=0.364 \times 10^{-11} \mathrm{~N}$ found at the same frequency in Fig. 6(a), thus ascribing this difference mainly to a quadrupole effect.

Similarly, at $f=25 \mathrm{MHz}$ the BEM approach gives a value for the second-order torque of $N_{z}^{(2)}=0.812 \times 10^{-16} \mathrm{Nm}$, whereas for the equivalent model we get analytically $N_{z}^{(2)}=$ $0.111 \times 10^{-16} \mathrm{Nm}$, which is almost an order of magnitude lower. The BEM value for the torque is again in good agreement with the difference, $\Delta N_{z}=0.843 \times 10^{-16} \mathrm{Nm}$, between the total torque and the dipolar approximation shown in Fig. 6(b).
The good agreements found for the second-order force and torque confirm that quadrupole effects are the main contribution to the frequency shifts of the DEP and ER spectra and that the effects from both the image charges on the electrodes and the contributions from higher order multipoles are much less significant. Finally, it is noticeable that the second-order contribution to the force calculated using the BEM method is more than 1 order of magnitude bigger than that provided by the equivalent model. These results clearly indicate that the use of an equivalent permittivity is a poor approximation when the cell is immersed in an electric field with significant nonuniformity, because the equivalent permittivity should be different for each multipolar moment.

\section{CONCLUSIONS}

We have calculated the dielectrophoretic force and electrorotational torque acting on a realistic four-shelled model of the yeast $S$. cerevisiae in the nonuniform rotating electric field generated by four coplanar square electrodes. For this purpose we have used the Maxwell stress tensor, regarded as the most rigorous approach for the derivation of field-induced forces. Then we have compared these results with numerical computations of the dipolar and quadrupolar contributions to the force and torque. To calculate the dipolar forces we did not follow the general method based on the Clausius-Mossotti factor of a homogeneous dielectric cell. Instead, we determined the complex polarizability of the actual yeast model directly from the internal field distribution. A comparison of the first-order dipolar forces and the total Maxwell stress forces shows the influence of all the higher order multipolar terms induced in the cell by the nonuniform fields. To isolate the second-order contribution we use a technique based on an integral equation for the polarization charge density, which has the additional numerical advantage of not being limited by the need of a very dense grid. This second order accurate contribution in turn is compared to the result obtained using 
the second-order Clausius-Mossotti factor with an equivalent homogeneous dielectric spherical model.

The comparison between the dipolar and the total forces and torques shows that the crossover frequencies of both DEP and ER spectra are shifted to higher values. The first-order DEP force is at least 1 order of magnitude larger than the second-order DEP force, and the frequency shifts observed in the DEP and ER spectra are mainly due to the second-order contributions. The comparison also shows that substituting the multilayer cell with an equivalent homogeneous permittivity model underestimates the second-order terms' contribution to the force and torque by factors of 12 and 7, respectively. This is an interesting observation of this analysis that should be taken into account when experimentally characterizing the electric properties of the cells by their frequency-dependent rotation.
To extract reliable cell information from DEP and ER spectra, good control of the cell position is required, the effects of multipolar contributions being more noticeable at low heights above the electrode plane and for wider electrode gaps.

We finally mention that although our realistic model of $S$. cerevisiae is spherical, the numerical methods we have used are readily applicable to more general cell shapes.

\section{ACKNOWLEDGMENTS}

The financial support of UCM-Santander to Bioelectromagnetism Research Group 910305, UCM-Santander Grant No. GR35/10-A-91056, and Comisión Interministerial de Ciencia y Tecnología Project No. FIS2008-00209 is gratefully acknowledged.
[1] R. Pethig, Biomicrofluidics 4, 022811 (2010).

[2] M. Mischel, A. Voss, and H. A. Pohl, J. Biol. Phys. 10, 223 (1982).

[3] W. M. Arnold and U. Zimmermann, J. Electrost. 21, 151 (1988).

[4] A. Alazzam, D. Roman, V. Nerguizian, I. Stiharu, and R. Bhat, Microfluid. Nanofluid. 9, 1115 (2010).

[5] A. K. Srivastava, M. Kim, S. M. Kim, M.-K. Kim, K. Lee, Y. H. Lee, M.-H. Lee, and S. H. Lee, Phys. Rev. E 80, 051702 (2009).

[6] J. P. Huang, K. W. Yu, G. Q. Gu, and M. Karttunen, Phys. Rev. E 67, 051405 (2003).

[7] S. Archer, H. Morgan, and F. J. Rixon, Biophys. J. 76, 2833 (1999).

[8] C. Dalton, A. D. Goater, J. P. H. Burt, and H. V. Smith, J. Appl. Microbiol. 96, 24 (2004).

[9] X. B. Wang, R. Pethig, and T. B. Jones, J. Phys. D 25, 905 (1992).

[10] X. B. Wang, Y. Huang, R. Holzel, J. P. H. Burt, and R. Pethig, J. Phys. D 26, 312 (1993).

[11] M. P. Hughes, X. B. Wang, F. F. Becker, P. R. C. Gascoyne, and R. Pethig, J. Phys. D 27, 1564 (1994).

[12] T. Sun, H. Morgan, and N. G. Green, Phys. Rev. E 76, 046610 (2007).

[13] M. P. Hughes, Phys. Med. Biol. 43, 3639 (1998).

[14] M. P. Hughes, S. Archer, and H. Morgan, J. Phys. D 32, 1548 (1999).

[15] J. Gimsa, R. Glaser, and G. Fuhr, in Physical Characterization of Biological Cells, edited by W. Schütt, H. Klinkmann, I. Lamprecht, and T. Wilson (Verlag Gesundheil GmbH, Berlin, 1991), pp. 295-323.

[16] R. Hölzel, J. Phys. D 26, 2112 (1993).

[17] H. P. Schwan, Ferroelectrics 86, 205 (1988).

[18] T. B. Jones, J. Electrost. 6, 69 (1979).

[19] R. Paul and K. V. I. S. Kaler, Phys. Rev. E 48, 1491 (1993).

[20] T. B. Jones, IEEE Eng. Med. Biol. Mag. 22, 33 (2003).

[21] X. J. Wang, X. B. Wang, and P. R. C. Gascoyne, J. Electrost. 39, 277 (1997).

[22] N. G. Green, A. Ramos, and H. Morgan, J. Electrost. 56, 235 (2002).

[23] D. F. Chen, H. Du, W. H. Li, and C. Shu, J. Micromech. Microeng. 15, 1040 (2005).
[24] C. H. Kua, Y. C. Lam, C. Yang, K. Youcef-Toumi, and I. Rodríguez, J. Electrost. 66, 514 (2008).

[25] T. B. Jones, Electromechanics of Particles (Cambridge University Press, Cambridge, 1995).

[26] U. Zimmermann and G. A. Nell, Electromanipulation of Cells (CRC Press, Boca Raton, FL, 1995).

[27] E. R. Mognaschi and A. Savini, J. Phys. D 16, 1533 (1983).

[28] Y. A. Chizmadzhev, P. I. Kuzmin, and V. P. Pastushenko, Biol. Membrany 2, 1147 (1985).

[29] V. P. Pastushenko, P. I. Kuzmin, and Y. A. Chizmadzhev, Stud. Biophys. 110, 51 (1985).

[30] V. P. Pastushenko, P. I. Kuzmin, and Y. A. Chizmadzhev, Biol. Membrany 5, 65 (1988).

[31] T. Schnelle, R. Hagedorn, G. Fuhr, S. Fielder, and T. Muller, Biochim. Biophys. Acta 1157, 127 (1993).

[32] F. A. Sauer, H. Frohlich, and F. Kremer, eds., Coherent Excitations in Biological Systems (Springer, Berlin, 1983).

[33] F. A. Sauer and R. W. Schlögl, in Interactions Between Electromagnetic Fields and Cells, edited by A. Chiabrera, C. Nicolini, and H. P. Schwan (Plenum, New York, 1985), pp. 203-252.

[34] J. Yang, Y. Huang, X. J. Wang, X. B. Wang, F. F. Becker, and P. R. C. Gascoyne, Biophys. J. 76, 3307 (1999).

[35] J. Gimsa, T. Muller, T. Schnelle, and G. Fuhr, Biophys. J. 71, 495 (1996).

[36] V. L. Sukhorukov and U. Zimmermann, J. Membr. Biol. 153, 161 (1996).

[37] Y. Huang, X. B. Wang, F. F. Becker, and P. R. C. Gascoyne, BBA Biomembranes 1282, 76 (1996).

[38] J. Gimsa, R. Glaser, and G. Fuhr, Stud. Biophys. 125, 71 (1988).

[39] K. Maswiwat, M. Holtappels, and J. Gimsa, Electrochim. Acta 51, 5215 (2006).

[40] K. Maswiwat, M. Holtappels, and J. Gimsa, ScienceAsia 33, 061 (2007).

[41] A. M. Woodward and D. B. Kell, Bioelectrochem. Bioener. 24, 83 (1990).

[42] M. D. Vahey and J. Voldman, Anal. Chem. 80, 3135 (2008).

[43] R. Hölzel, Biophys. J. 73, 1103 (1997).

[44] E. Vitols, R. J. North, and A. W. Linnane, J. Biophys. Biochem. Cytol. 9, 689 (1961). 
[45] C. W. Moore, R. del Valle, J. McKoy, A. Pramanik, and R. E. Gordon, Antimicrob. Agents Ch. 36, 2497 (1992).

[46] J. Mulholland, D. Preuss, A. Moon, A. Wong, D. Drubin, and D. Botstein, J. Cell Biol. 125, 381 (1994).

[47] J. L. Sebastián, S. Muñoz, M. Sancho, and G. Álvarez, Phys. Rev. E 78, 051905 (2008).

[48] E. Prodan, C. Prodan, and J. H. Miller Jr., Biophys. J. 95, 4174 (2008).
[49] A. Di Biasio and C. Cametti, Phys. Rev. E 82, 021917 (2010).

[50] A. Di Biasio, L. Ambrosone, and C. Cametti, Phys. Rev. E 82, 041916 (2010).

[51] M. Sancho, G. Martínez, and C. Martín, J. Electrost. 57, 143 (2003).

[52] M. Sancho, G. Martínez, S. Muñoz, J. L. Sebastián, and R. Pethig, Biomicrofluidics 4, 022802 (2010).

[53] D. B. Geselowitz, Biophys. J. 7, 1 (1967). 